\title{
Analysis Method of Calculation Parameters of Heat and Mass Transfer Processes in the Stirling Engine
}

\author{
Sergej Semyonov, Sergej Tikhonov, Mihail Donchenko, \\ Jurij Lukyanov, Andrej Perminov \\ Pskov State University, Faculty of Engineering and Construction Technologies, Pskov State University, \\ Faculty of Computer Science and Power Engineering. \\ Address: Russia, g. Pskov, pl. Lenina, d. 2, 180000.
}

\begin{abstract}
The article highlights the optimal calculation methods for determining the parameters of heat and mass transfer processes occurring in the rotary-vane engine with an external supply of heat. It is shown that the mathematical model of working processes must consist of two parts. One part describes the processes occurring in the isolated volume. The second part describes the processes of mass exchange between the working chambers of two modules, as well as a heater or a cooler.
\end{abstract}

Keywords: rotary-vane, heat and mass transfer processes, expiration process, expansion and contraction, nodal analysis methods, Stirling thermodynamic cycle, analysis of methods of calculation.

\section{INTRODUCTION}

In recent years greatly increased interest in engines with an external supply of heat, which is associated with the possibility of transformation in these engines in the energy of any kind of heat and low levels of toxicity, which significantly expands the scope of their application compared to the most common currently types of engines (engines of internal combustion, diesel engines). In most cases objects of interest are not the piston engines working on a thermodynamic cycle of Stirling, but rotary engine thermodynamic cycle that differs from a thermodynamic cycle of Stirling a little.

Rotary engines have a number of significant advantages over piston engines, as evidenced by a large number of scientific articles and patents devoted to this subject [1]. One of such engines is a rotaryvane engine with an external supply of heat (RVE), developed the staff of the Pskov State University [2, $3]$. Engine unites the advantages of the rotary scheme and the external heat supply. RVE can be a substitute for traditional types of energy unit in areas such as autonomous systems of power supply, including cogeneration unit, electric generators of low power, solar power unit.

Experience designing of a heat engines shows that the creation of adequate experimentally verified mathematical models of processes occurring in the engine can significantly reduce the amount of costly experimental studies. We can say that the creation of an adequate mathematical model is one of the keys to success engine development. The aim of the article is to determine the optimal method of calculation to determine the parameters of heat and mass transfer processes occurring in RVE.

\section{BRIEF DESCRIPTION OF THE THERMODYNAMIC CYCLE}

RVE consists of two modules. The two modules have a similar structure and are rotated relative to each other at an angle of $45^{\circ}$. RVE scheme is shown in Figure 1.

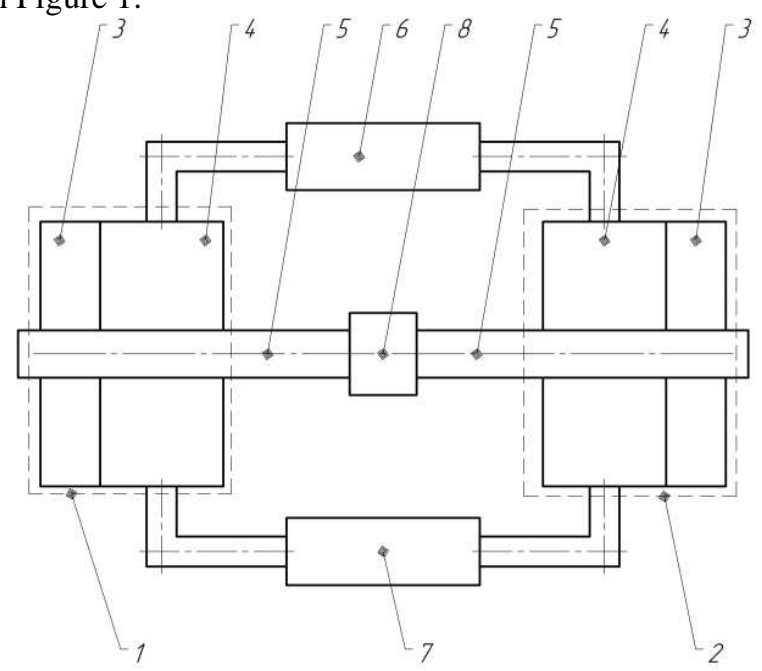

Fig. 1. RVE scheme:

1 - 1st module; 2 - 2st module; 3 - transformation mechanism; 4 Vanes group; 5 - output shaft; 6 - heater; 7 - cooler; 8 - coupling.

Vanes group has four working chambers of variable volume. In everyone working chamber http://dx.doi.org/10.17770/etr2017vol3.2513 
occurs thermodynamic cycle, which is not much different from the thermodynamic cycle of Stirling. Changing the angle between the axes of the pistons $\psi$ and the volume of the working chambers, depending on the output shaft angle $\alpha$ is due to the rotation of the pistons position 2 figure 2 and position 3 figure 2 with different angular velocity. Pistons position 2 figure 2 mounted on the outer rotor have an angular velocity $\omega_{1}(\alpha)$. Pistons position 3 figure 2 mounted on the internal rotor have angular velocity $\omega_{2}(\alpha)$.

General view of the vanes group RVE is shown in Figure 2.

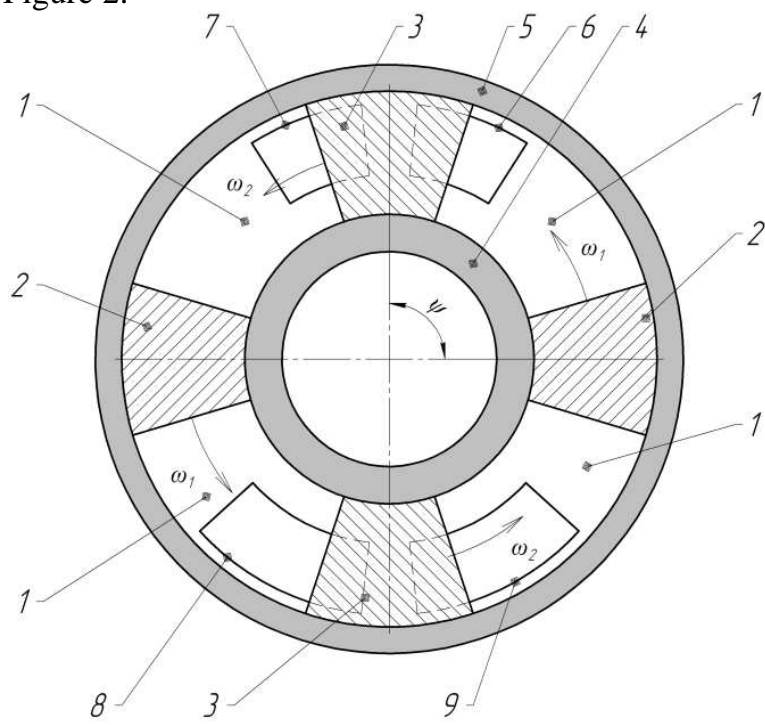

Fig. 2. A general view of a vanes group:

1 - working chamber; 2,3 - piston; 4 - rotors; 5 - housing; 6 exhaust port heater; 7 - inlet port heater; 8 - exhaust port cooler: 9 - inlet port cooler.

During one revolution of the output shaft in everyone working chamber of the engine is carried out one complete thermodynamic cycle. Thermodynamic cycle consists of the following processes:

- compression of the working fluid in the isolated volume;

- release of the working fluid through a heater in another module;

- inlet of the working fluid through the heater of another module;

- expansion of the working fluid in the isolated volume;

- release of the working fluid through the cooler in the other module;

- inlet of the working fluid through the cooler from another module.

The thermodynamic cycle that takes place in RVE is closed over the entire engine and opened with respect to one working chamber. The processes of expansion and contraction of the working fluid occurs in isolation. The processes of intake and exhaust of the working fluid occurs with a compound of the working chambers of two modules with a heater or cooler.

Since in every working chamber RVE passes sequentially through the six thermodynamic processes, some of which takes place in an isolated volume, and some with the release of the working fluid into the working chamber of another module through the heater or cooler, for each of these processes is necessary to develop separate mathematical models that will determine the main parameters of the working fluid (temperature, pressure, weight, etc.) during each processes of cycle, as a function of the angle of rotation of the output shaft, and then to unite model to determine the parameters for all cycle times.

\section{IDENTIFICATION OF METHODS FOR CALCULATING THE PARAMETERS OF THE PROCESS OF EXPANSION AND CONTRACTION OF THE WORKING FLUID}

The scheme of calculating the expansion and compression processes that occur in an isolated volume is shown in Figure 3.

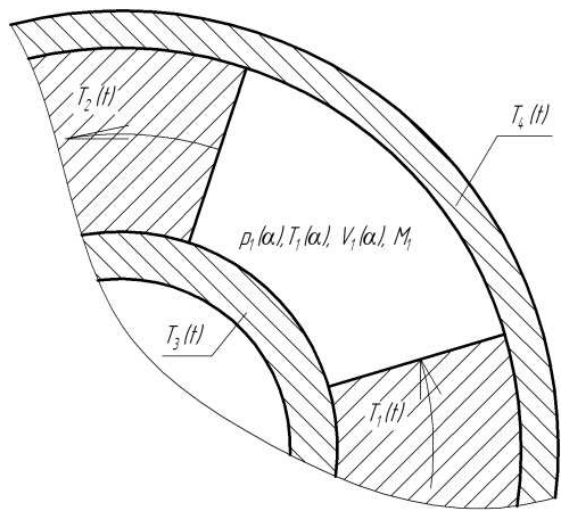

Fig. 3. The scheme of calculating processes of expansion and contraction

In accordance with [4] processes that take place in an isolated volume, described by the equation of the first law of thermodynamics constant weight (1):

$$
M C_{V} d T+p d V+d Q_{w}=0,
$$

where $M-$ the mass of the working fluid in the chamber; $C_{V}-$ specific isochoric heat capacity working fluid; $T$ - working fluid temperature in the chamber; $d Q_{w}$ - heat given (received) the working fluid as a result of heat exchange with the chamber walls; $p$-pressure; $V$-volume.

After a series of transformations of the equation (1) is obtained by two differential equations (2) and (3).

$$
\frac{d T}{d \alpha}=\frac{(k-1) T}{V} \frac{d V}{d \alpha}-\frac{1}{M C_{V}} \frac{d Q_{w}}{d \alpha}
$$




$$
\begin{array}{r}
\frac{d p}{d \alpha}=\frac{k p}{V} \frac{d V}{d \alpha}-\frac{p}{M T C_{V}} \frac{d Q_{w}}{d \alpha} \\
\text { Item } \frac{d Q_{w}(\alpha)}{d \alpha} \text { in accordance with [5] equals: } \\
\frac{d Q_{w}(\alpha)}{d \alpha}=\sum_{i=1}^{n} \frac{\beta_{i} F_{i}\left(T(\alpha)-T_{w_{i}}\right)}{\omega}
\end{array}
$$

where $\beta_{i}-$ the average value of heat transfer coefficient surface separate part; $F_{i c}$ - heat exchange surface area separate part; $T_{w i}$ - surface temperature separate part; $\mathrm{n}$ - number of surfaces, forming a working chamber; $i$ - serial number surface forming a working chamber.

In preliminary calculations, the heat transfer to the walls it is possible to neglect $\left(d Q_{w}=0\right)$ and considered as adiabatic process. For a complete description of the processes of heat and mass transfer during the expansion and compression cycles need to solve equations (2) and (3) for the start of the engine and steady operation engine. At the time of starting the engine wall temperature is $293 \mathrm{~K}$. In the case of the steady operation of the engine for each of the details that form the working chamber should be decided stationary heat conduction differential equation and defined wall temperature $T_{w i}$. Then it will be possible to determine the heat which is transferred from the working fluid to the walls.

Thus, the processes of expansion and compression of working fluid in the isolated volume may be determined by analytical methods with sufficient accuracy. Numerical study of the processes of expansion and contraction of the working body with sufficient accuracy can be made in systems of computer mathematics (for example, MATLAB, Mathcad, Maple, Mathematica). The results of calculations of the processes of expansion and contraction in computer mathematics system Mathcad are given in [6].

\section{ANALYSIS OF THE MATHEMATICAL MODELS USED TO CALCULATE THE HEAT AND MASS TRANSFER PROCESSES IN PISTON ENGINES STIRLING}

Analysis cycles of the supply and removal of heat that occur in piston Stirling engines, has shown their considerable similarity with the cycles of the mass transfer in RVE that allows them to be used as a basis for the development of a methodology of calculation. The main difference is the presence in the construction of the piston Stirling engine regenerator. It may be noted that the mathematical description of mass transfer processes characterized by considerable complexity, as evidenced by the fact that the first Stirling engine was created in 1816, and the mathematical description of its work has been developed only in 1871.

All the basic mathematical models, which are used to calculate the piston Stirling engines, can be divided into four groups.

- Workflow model of the first order.

These models are used in the preliminary analysis of the piston Stirling engines. Motor parameters are determined on the basis of general relations, which are based on experimental studies. Known ratio to assess the capacity of the Stirling engine, named Bill ratio [7]:

$$
P=Z \beta_{E} V_{S P} p_{c p} N / 6000
$$

$P$ - shaft power; $Z$ - the number of individual thermodynamic cycles; $\beta_{E}-$ Bill number, determined by the formula $\beta_{E}=0,034-0,052 \xi$, where $\xi$ - the ratio of the refrigerator temperature to the heater temperature; $V_{S P}-$ a working volume of the compression chamber; $p_{c p}$ - average pressure of the cycle; $N$ - rotational speed.

All found in the literature approximate relations obtained for the piston Stirling engines and their application for the analysis RVE work seems inappropriate, as is significantly different not only engine design, but also the thermodynamic cycle, which implements by the engine.

- Workflow model of the second order.

These models are based on analysis thermodynamic cycle flowing into the engine cylinders, without using heat and mass transfer laws and allow determining the temperature and pressure of the working fluid as a function of the angle of rotation of the engine output shaft. Typically, these models are based on a mathematical model isothermal [6]. This model assumes that the expansion and contraction cycles run at a constant temperature of the working fluid, which is achieved due to supply or remove heat.

The basic admissions of these models are as follows:

- the absence of leaks of the working fluid,

- simplification of heat transfer processes of the working fluid with the walls;

- working fluid temperature in the heater and cooler, equal respectively, is cooler temperature and heater temperature.

Accounting for these factors produced by introducing empirical correction factors.

Workflow model of the second order are used in the preliminary stages of design and allow us to estimate the temperature and pressure in the working chamber of the engine, the output power of the engine and its dimensions. These methods do not allow to accurately determining the parameters of the engine 
working process, the error of the results may be around $30-40 \%$. Their main purpose to determine the maximum achievable engine performance. With respect to the piston Stirling engine of the second order model developed by Schmidt, Martini, Finkelstein, Rios, Fejer and others.

- Models workflows third order (nodal analysis methods).

The first study of processes that occur in the piston Stirling engines, method nodal analysis was carried out in 1975, Finkelstein. In models of nodal analysis jointly modeled energy transfer processes and the working fluid flow. To do this, the engine is divided into several sections, for this sections make up and solve a system of ordinary differential equations consisting of the laws of conservation of mass, momentum and energy. The equations are solved by numerical methods, the most common of which is the method of Runge-Kutta methods.

Nodal methods, in contrast to the methods of the first and second order, allow taking into account a number of important factors affecting the operation of the engine. These factors include unsteady heat transfer from the heater and cooler, heat transfer to the walls of the working chamber of the engine. At the same time, the methods of nodal analysis does not take into account the geometric characteristics of each node and the working parameters of the node are considered lumped and depend only on the time.

For piston Stirling engines are best known nodal model workflows Finkelstein, Uriel and the Organ.

As a basis for mathematical model of mass transfer processes in the RVE can be used model, which describes the workflows in the piston Stirling engine with rhombic drive. In this model, the core engine is divided into 13 units. For each of the resulting nodes need to record two ordinary differential equations: the equation of the first law of thermodynamics variable mass (6) and the law of conservation of mass (7):

$$
\begin{gathered}
\frac{d}{d t}\left(M C_{V} T\right)=h A\left(T_{w}-T\right)+ \\
c_{p}\left(w_{i} T_{i}-w_{0} T_{0}\right)-p \frac{d V}{d t} \\
\frac{d V}{d t}=G_{i}-G_{0}
\end{gathered}
$$

where $h$ - the working fluid heat transfer coefficient; $c_{p}$ - isobaric specific heat of the working fluid; $G_{i}$ - mass flow rate working fluid entering the chamber; $G_{0}$ - mass flow rate of the working fluid flowing out of the chamber; $T_{i}$ - the temperature of the working fluid entering the chamber; $T_{0}-$ the temperature of the working fluid out the chamber.
The system of ordinary differential equations must be supplemented by the equation of state of an ideal gas (8):

$$
p V=M R T
$$

Equations (6) and (7), (8), which are prepared for each of the volumes 13, are solved together, whereby determining pressure, temperature and mass of the working fluid 13 in each of the volumes.

- Computer modeling of the finite element method and finite volume method.

These methods are the most accurate of all the above methods, since they allow taking into account the heat exchange fluid with the walls, geometry of the working chamber, the influence of the intake and exhaust tracts, as well as transients. Also, they allow you to take into account for the flow of the working fluid effects of turbulence and local resistance. To apply these methods require special computer programs (Star CCM +, Flowvision, Ansys Fluent and others). In most cases, these methods are used in the final design stages, together with experimental studies, or instead of them, when you need to calculate the parameters of working processes already designed motor.

\section{IDENTIFICATION OF METHODS FOR CALCULATING THE PARAMETERS OF MASS TRANSFER PROCESSES IN RVE}

After analyzing the advantages and disadvantages of all basic mathematical models, which are used for the calculation of piston engines, we can conclude that the mathematical model of mass transfer processes in the RVE must be carried out on the basis of nodal analysis methods that provide sufficient accuracy of calculations, and are much smaller in comparison with the methods of computer modeling requirements to computing resources.

The main nodes (control volume), in which will be determined by the parameters of the working body, it is proposed to use the following: The working chamber of the 1st module, inlet manifold the heater or the cooler, tube bundle, and an outlet manifold the heater or the cooler, working chamber 2 nd module.

To determine the mass transfer parameters for each node must be written three equations: the equation of the first law of thermodynamics variable mass equation of second mass flow rate and the equation of state [8]:

$$
\left\{\begin{array}{l}
\sum_{i M_{i s t}} i d M_{i s t}=d(M u)+p d V+d Q_{W} \\
d t \\
p V=M R T
\end{array}\right.
$$

where $i$ - enthalpy of the working fluid flowing into the control volume or arising from it; $d M_{i s t}$ elementary mass of the working fluid flowing into the control volume or arising from it; $u$ - the internal 
energy of the working fluid; $\mu$ - expenditure coefficient; $\rho$ - the working fluid density; $S$ - flow section area.

Thus, to describe the parameters of mass transfer processes in the RVE it is necessary to solve differential-algebraic system consisting of 15 equations.

Presentation of the tube bundle as a node with lumped parameters is a clear simplification and can make a significant error in the calculation results, therefore it is necessary to consider separately the processes in the tube bundle and introduce correction factors in the equations (9), if necessary. In general, the processes in the tube bundle described by a system of the 4 equations: the equation of state, the momentum equation, continuity equation and the energy equation.

Determination of expenditure rate $\mu$, which is included in the system of equations (9), is a complex hydrodynamic task that is complicated by the nonstationary processes occurring in RVE. This problem can be solved by experimental methods or by computer simulation.

The proposed scheme for calculating the parameters of mass transfer process is shown in Figure 4.

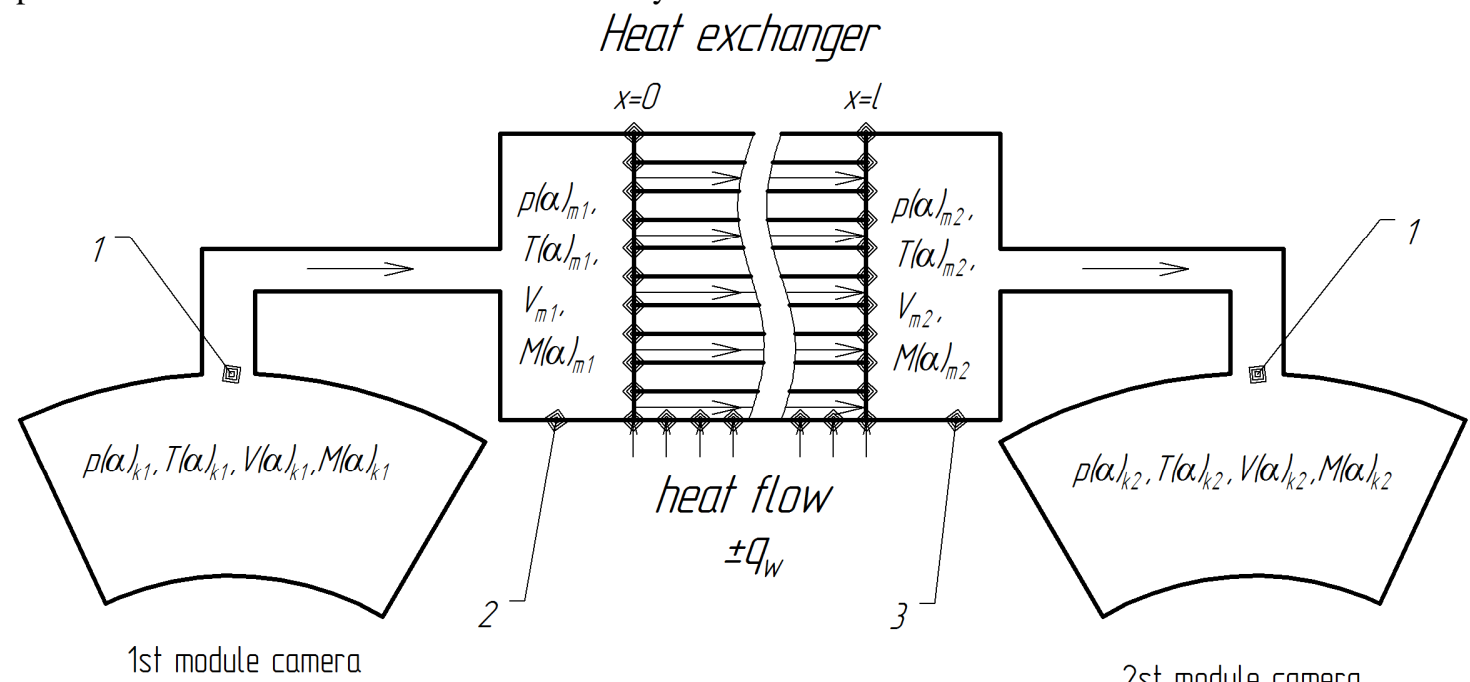

Fig. 4 - Diagram of calculation workflows RVE:

1 - inlets and outlets of the working chamber; 2 inlet manifold; 3 - outlet manifold.

$p(\alpha)_{k 1}, T(\alpha)_{k 1}, V(\alpha)_{k 1}, M(\alpha)_{k 1}$ - parameters of the working fluid in the working chamber 1st module; $p(\alpha)_{m 1}$, $T(\alpha)_{m 1}, V(\alpha)_{m 1}, M(\alpha)_{m 1}$ - parameters of the working fluid in the inlet manifold; $p(\alpha)_{m 2}, T(\alpha)_{m 2}, V(\alpha)_{m 2}$, $M(\alpha)_{m 2}$ - parameters of the working fluid in the outlet manifold; $p(\alpha)_{k 2}, T(\alpha)_{k 2}, V(\alpha)_{k 2}, M(\alpha)_{k 2}-$ parameters of the working fluid in the 1 st module.

Numerical study of the mass transfer processes of the working body is performed in computer mathematics systems (for example, MATLAB, Mathcad, Maple, Mathematica). In the future it is planned to check the adequacy of the assumptions made in the mathematical model to analyze the mass transfer processes of the working body. The check can be carried out by finite element or finite volume methods in special programs (Star CCM +, Flowvision, Ansys Fluent and others).

\section{CONCLUSION}

Carried out in this article, the analysis showed that the parameters of the mathematical model of heat exchange and mass transfer processes occurring in the RVE, should consist of two parts. One part describes the processes taking place in an isolated volume based on the differential equations (2) and (3). The second part describes the process of mass transfer based on the system of differential-algebraic equations (9). The mathematical model includes the following key assumptions: no leakage of the working fluid from the working chamber of the engine; workflow settings in the control volumes are concentrated; the working fluid flow processes are considered quasi-stationary. Built on the basis of recommendations given in this paper, the mathematical model of heat and mass transfer processes in the RVE will significantly reduce the amount of experimental research.

\section{REFERENCES}

[1] Grinjov D.V. Structural schemes and principles of rotary vane machines // Vestnik PskovGU. The economic and technical sciences. Vol. 5. Pskov Publishing house PskovGU, 2014, 142-150.

[2] RF Patent 2374526 for an invention. Int. Cl. F16H25/04. Mechanism for converting motion / Lukyanov Y.N., Zhuravlev Y.N. et al. Publ. 27.11.2009. Bull. Number 33.

[3] RF Patent 2387844 for an invention. Int. Cl. F01G1/077, F02G1/044. Rotary-vane engine with an external supply of heat / Lukyanov Y.N., Zhuravlev Y.N. et al. Publ. 27.04.2010. Bull. Number 12. 
[4] Laushkina L.A., Solohina G.Je., Cherkasova M.V. Practical Course in Physics. Molecular Physics andThermodynamics Ed. prof. GG Spirin. A manual for schools. M.: VVIA im. prof. N.E. Zhukovskogo, 2008, 156

[5] Tsvetkov F.F., Grigoriev B. A. Heat and Mass Transfer: A manual for schools. - 2nd ed., Rev. and add. M: Publishing House of the MEI, 2005, 550.
[6] Semyonov S.N. Calculation of temperature and pressure in the rotary-vane engine with external heat supply // Vestnik PskovGU. The economic and technical sciences. Vol. 5. Pskov Publishing house PskovGU, 2014, 170-176.

[7] Reader T., Hooper C. Stirling Engines. M.: Mir, 1986, 464.

[8] Petrychenko R.M. Physical basis intracylinder processes in internal combustion engines. L.: Publishing House of Leningrad. University, 1983, 244. 